\title{
Guidelines for NHL: Updates to the Management of Diffuse Large B-Cell Lymphoma and New Guidelines for Primary Cutaneous CD30+ T-Cell Lymphoproliferative Disorders and T-Cell Large Granular Lymphocytic Leukemia
}

Presented by Andrew D. Zelenetz, MD, PhD

\begin{abstract}
During his presentation at the NCCN 19th Annual Conference, Dr. Andrew D. Zelenetz reviewed the updates to the 2014 NCCN Clinical Practice Guidelines in Oncology (NCCN Guidelines) for NonHodgkin's Lymphomas. Dr. Zelenetz first discussed the updates for diffuse large B-cell lymphoma (DLBCL), focusing primarily on the emergence of MYC-positive DLBCL; the limited role of imaging in early-stage disease; new treatment options; the challenge of tumor heterogeneity; and the impact of cell of origin in the selection of future therapies. Then, on behalf of Dr. Steven Horwitz, Dr. Zelenetz presented the new guidelines for primary cutaneous $\mathrm{CD} 30+$ T-cell lymphoproliferative disorders and T-cell large granular lymphocytic leukemia. (J Nat/ Compr Canc Netw 2014;12:797-800)
\end{abstract}

\footnotetext{
"We are rapidly moving beyond R-CHOP for all patients with diffuse large B-cell lymphoma [DLBCL]," declared Andrew D. Zelenetz, MD, PhD, Professor of Medicine, Weill Medical College of Cornell University; Vice Chair, Medical Informatics, Memorial SloanKettering Cancer Center, New York; and Chair of the NCCN Non-Hodgkin's Lymphomas (NHL) Panel. Tar-

Presented by Andrew D. Zelenetz, MD, PhD, Professor of Medicine, Weill Medical College of Cornell University, and Vice Chair, Medica Informatics, Department of Medicine, and Attending Physician in the Lymphoma Service, Memorial Sloan-Kettering Cancer Center, New York, New York.

Dr. Zelenetz has disclosed that he receives grant or research support from Genentech, Inc., GlaxoSmithKline, and Roche Laboratories, Inc., and consulting fees or honoraria from Celgene Corporation, Cephalon, Inc., GlaxoSmithKline, sanofi-aventis US, and Seattle Genetics. He also serves as a scientific advisor for Cancer Genetics, and Gilead.

Correspondence: Andrew D. Zelenetz, MD, PhD, Memorial SloanKettering Cancer Center, 1275 York Avenue, Box 330, New York, NY 10065. E-mail: zeleneta@mskcc.org
}

geted agents interact with specific pathways, necessitating patient selection based on cells of origin, mutation status, or both, he noted.

\section{Updates to the NCCN Guidelines for DLBCL}

\section{Diagnosis and Imaging}

The immunohistochemistry (IHC) panel was modified to include the MYC expression, which is now considered essential for the diagnosis of DLBCL. In addition, the guidelines also highlight the need to perform fluorescence in situ hybridization (FISH) to identify MYC rearrangements in selected patients, Dr. Zelenetz pointed out. "This change in the workup is not just for pathologists," explained Dr. Zelenetz, "but it has important clinical relevance."

About $5 \%$ to $10 \%$ of DLBCLs harbor a MYC rearrangement, which is associated with a poor outcome. ${ }^{1}$ Over the past few years, emerging data suggest that DLBCL with concurrent MYC and BCL2 gene rearrangements (referred to as double-hit lymphomas, occurring in approximately $6 \%$ of patients with DLBCL) is associated with a particularly poor outcome. ${ }^{2}$ More recent data have shown that concurrent expression of MYC and BCL2 proteins (which occurs in approximately $30 \%$ of patients with DLBCL) as determined by IHC is also a strong predictor of poor outcome in patients with DLBCL treated using R-CHOP (rituximab plus cyclophosphamide, doxorubicin, vincristine, prednisone). ${ }^{3}$ "There is no evidence-based standard of care for these patients and they really need to go on prospective 
clinical trials," Dr. Zelenetz emphasized. Inhibitors of MYC expression that are in early phases of development may play an important role in the treatment of patients with double-hit lymphomas.

Turning to imaging, Dr. Zelenetz said, "We revisited and keep revisiting the role of imaging in DLBCL as new data emerge." In terms of follow-up imaging for patients with early-stage disease who experience a complete response after completion of induction chemotherapy, the updated guidelines now state that repeat CT scanning should not be performed routinely but only as clinically indicated. This change in the guidelines reflects the limited utility of surveillance imaging, particularly in patients with localized disease, he added.

\section{Current and Future Treatment Options}

Optimal first-line therapy is more controversial with primary mediastinal B-cell lymphoma (PMBL) than with other subtypes of NHL, and is so stated in the updated 2014 NCCN Guidelines for NHL. However, the guidelines include 3 different options for PMBL. R-CHOP followed by radiation therapy (RT) and DA-EPOCH-R (dose-adjusted etoposide, doxorubicin, cyclophosphamide, vincristine, prednisone and rituximab) are included with a category $2 \mathrm{~A}$ recommendation. R-CHOP followed by ICE (ifosfamide, carboplatin, etoposide) with or without RT is included with a category $2 \mathrm{~B}$ recommendation, although Dr. Zelenetz admitted that there are no data to suggest a benefit with RT.

In a phase II study of 67 patients with PMBL, DAEPOCH-R produced an event-free survival rate of $93 \%$ and an overall survival rate of $97 \%$ at a median followup of 5 years. ${ }^{4}$ Dr. Zelenetz acknowledged that these results are promising, but because the study had only 67 patients, more data are needed to recommend DAEPOCH-R as standard of care for patients with PMBL.

For patients with poor left ventricular function, the updated guidelines now include the attenuated immunotherapy regimen known as $\mathrm{R}$-mini-CHOP (decreased dose of CHOP and a conventional dose of rituximab) as an option for patients older than age 80 with comorbidities ("very frail patients"), based on the encouraging progression-free and overall survival results reported by Peyrade et al. ${ }^{5}$ "These were quite good curves in a group where the minimum age was 80 ," stated Dr. Zelenetz.

Morphologic heterogeneity represents a therapeutic challenge in DLBCL. Gene expression profiling has identified at least 3 subtypes: germinal cen- ter B-cell (GCB) DLBCL, activated B-cell (ABC) DLBCL, and PMBL. ${ }^{6}$ An immunohistochemical model incorporating CD10, BCL6, and IRF4/MUM1 has also been developed to distinguish the GCB and non-GCB subtypes.? "Even in the rituximab era, we have not been able to overcome this tumor heterogeneity," admitted Dr. Zelenetz. "Furthermore, the molecular pathways within the subtypes of GCB and non-GCB DLBCL are quite different." For example, EZH2 gene mutations are restricted to GCB subtype and are found in about $22 \%$ of DLBCL. ${ }^{8}$ Small-molecule EZH2 inhibitors that are currently in clinical trials can block the proliferation of and kill cell lines with activating EZH2 gene mutations.

The cells of origin may play an integral role in guiding the selection of future therapies for DLBCL. Dr. Zelenetz focused on the emerging data on the use of lenalidomide for non-GCB subtype ${ }^{9}$ and ibrutinib (Bruton tyrosine kinase inhibitor) for the ABC subtype of relapsed/refractory DLBCL. ${ }^{10}$

In a retrospective review of 44 patients with relapsed/refractory DLBCL, a significant difference in clinical response to lenalidomide was found in those with non-GCB versus GCB subtype. ${ }^{9}$ In fact, the overall response rate was a "miserable" $9 \%$ in GCB subtype but more than 50\% in non-GCB subtype. The combination of lenalidomide and R$\mathrm{CHOP}$ is being evaluated in several ongoing studies.

The use of ibrutinib for relapsed/refractory DLBCL was assessed in a phase II study of 70 patients. ${ }^{10}$ The investigators demonstrated that ibrutinib yielded a clinically meaningful response rate in those with the ABC subtype but not in other molecular subtypes (41\% vs 23\%). Ongoing studies are comparing the use of R-CHOP with and without ibrutinib, specifically in patients with non-GCB DLBCL.

Dr. Zelenetz concluded that lenalidomide and ibrutinib appear to overcome the adverse impact of non-GCB DLBCL, although intensive conventional chemotherapy may achieve the same results at a lower cost. However, the results are not yet mature enough to be incorporated into the NCCN Guidelines.

\section{New Guidelines in T-Cell Lymphomas}

\section{Primary Cutaneous CD30+ T-Cell Lymphoproliferative Disorders}

The cutaneous lymphomas are generally a complex admixture of slow-growing T-cell diseases, relatively 
indolent B-cell diseases, and precursor lesions. The new guidelines for primary cutaneous CD30+ T-cell lymphoproliferative disorders provide recommendations for the treatment of primary cutaneous CD30+ anaplastic large cell lymphoma (ALCL) and lymphomatoid papulosis (LyP). The differential diagnosis of $\mathrm{CD} 30+$ lymphoid infiltrates in the skin can range from benign, reactive processes to malignant neoplasms. "An arthropod reaction can look just like LyP," remarked Dr. Zelenetz, so clinicopathologic correlation is essential. "The expression of CD30 is not a prognostic marker for good or bad results," he revealed.

It is necessary to distinguish between primary cutaneous CD30+ ALCL and LyP, because both have similar immunophenotypes. "LyP is at one end of the spectrum, and primary cutaneous CD30+ ALCL is at the other end," explained Dr. Zelenetz, "but many of the presentations are in between." Characteristics of LyP include $100 \%$ spontaneous regression, papular lesions less than $1 \mathrm{~cm}$, and crops of lesions, whereas traits of cutaneous CD30+ ALCL include less frequent spontaneous regression, larger or deeper lesions, and multifocal lesions that may present alone, in a group, or in a cluster. The outcome for patients with LyP and primary cutaneous ALCL "is very good," with 10-year disease-specific survival rates of $100 \%$ and $96 \%$, respectively. ${ }^{11}$ The exception to the rule is primary cutaneous ALCL with extensive limb disease. This more aggressive subtype is generally refractory to local RT and systemic therapies and tends to have a poorer outcome. ${ }^{12}$

The first step in the treatment algorithm for primary cutaneous CD30+ T-cell lymphoproliferative disorders is to distinguish them primarily from CD30+ transformed mycosis fungoides. The treatment for patients with LyP depends on the presence or absence of symptoms. Observation is preferred for asymptomatic patients with limited lesions. "Don't overtreat these tumors," emphasized Dr. Zelenetz. "Spontaneous remission is extremely common, and the death rate in our experience with LyP is zero." For patients who are symptomatic with extensive lesions, methotrexate, phototherapy, systemic retinoids, and topical steroids are listed as treatment options. "Topical steroids are effective but not great," he admitted, and there is limited experience with bexarotene.

As for primary cutaneous ALCL, "it must be distinguished from the skin presentation of systemic ALCL," Dr. Zelenetz pointed out. Primary cutane- ous ALCL is characterized by skin-only presentation with or without the involvement of regional nodes, and indolent disease course, with frequent skin relapses but rare progression to extracutaneous sites. "Treatment depends on presentation," stated Dr. Zelenetz. Surgical excision with or without local RT is indicated for patients with solitary lesions, whereas methotrexate is the preferred treatment for those with multifocal lesions. RT, systemic retinoids, pralatrexate, and observation (if asymptomatic) are also included as options.

\section{T-Cell Large Granular Lymphocytic Leukemia}

T-cell large granular lymphocytic leukemia (TLGLL) is another rare disease with a typically indolent course, accounting for between $2 \%$ and 5\% of chronic leukemias, according to Dr. Zelenetz. It tends to be associated with autoimmune disorders, ${ }^{13}$ particularly rheumatoid arthritis, and may be linked with B-cell malignancies.

In the NCCN Guidelines, peripheral blood smear analysis for cytology, flow cytometry on peripheral blood, bone marrow aspirate and biopsy, and adequate immunophenotyping are required to establish a diagnosis of TLGLL. "A trained medical pathologist and flow cytometrist are needed to interpret these results appropriately," revealed Dr. Zelenetz. And, it is important to do a workup for rheumatologic disorders.

As with other indolent lymphomas, treatment for TLGLL should be initiated only when indicated. Indications for therapy for TLGLL include severe neutropenia (absolute neutrophil count $<0.5 \mathrm{x}$ $10^{9} / \mathrm{L}$ ), moderate neutropenia with recurrent infections, symptomatic or transfusion-dependent anemia, autoimmune disorders associated with TLGLL, and B symptoms. In the absence of indications for treatment, a watch-and-wait strategy is reasonable. "Intervening early does not change the natural history of the disease," noted Dr. Zelenetz. For patients requiring treatment, first-line options include methotrexate, cyclophosphamide, and cyclosporin A, with or without corticosteroids. Although all of these options have reasonable activity and some have resulted in durable responses, results are based largely on retrospective, single-institution experiences involving a limited number of patients. ${ }^{14}$ Alemtuzumab has also been studied in patients with refractory disease, resulting in an overall response rate of nearly $60 \%$ and a duration of response ranging from 6 to 
24 months. ${ }^{14}$ Finally, Dr. Zelenetz indicated that a response may not be seen for several weeks, and as long as the treatment is tolerated without any side effects it should be continued.

\section{References}

1. Savage KJ, Johnson NA, Ben-Neriah S, et al. MYC gene rearrangements are associated with a poor prognosis in diffuse large B-cell lymphoma patients treated with R-CHOP chemotherapy. Blood 2009;114:3533-3537.

2. Johnson NA, Savage KJ, Ludkovski O, et al. Lymphomas with concurrent BCL2 and MYC translocations: the critical factors associated with survival. Blood 2009;114:2273-2279.

3. Green TM, Young KH, Visco C, et al. Immunohistochemical double-hit score is a strong predictor of outcome in patients with diffuse large B-cell lymphoma treated with rituximab plus cyclophosphamide, doxorubicin, vincristine, and prednisone. J Clin Oncol 2012;30:3460-3467.

4. Dunleavy K, Pittaluga S, Maeda LS, et al. Dose-adjusted EPOCHrituximab therapy in primary mediastinal B-cell lymphoma. N Engl J Med 2013;368:1408-1416.

5. Peyrade F, Jardin F, Thieblemont C, et al. Attenuated immunochemotherapy regimen (R-miniCHOP) in elderly patients older than 80 years with diffuse large B-cell lymphoma: a multicenter, single-arm, phase 2 trial. Lancet Oncol 2011;12:460468.

6. Alizadeh AA, Eisen MB, Davis RE, et al. Distinct types of diffuse large B-cell lymphoma identified by gene expression profiling. Nature 2000;403:503-511.
7. Hans CP, Weisenburger DD, Greiner TC, et al. Confirmation of the molecular classification of diffuse large B-cell lymphoma by immunohistochemistry using a tissue microarray. Blood 2004;103:275-282.

8. McCabe MT, Ott HM, Ganji G, et al. EZH2 inhibition as a therapeutic strategy for lymphoma with EZH2-activating mutations. Nature 2012;492:108-112.

9. Hernandez-Ilizaliturri FJ, Deeb G, Zinzani PL, et al. Higher response to lenalidomide in relapsed-refractory diffuse large B-cell lymphoma in nongerminal center B-cell-like than in germinal center B-cell-like phenotype. Cancer 2011;117:5058-5066.

10. Wilson WH, Gerecitano JF, Goy A, et al. The Bruton's tyrosine kinase inhibitor, ibrutinib, has preferential activity in the $\mathrm{ABC}$ subtype of relapsed/refractory de novo diffuse large B-cell lymphoma: interim results of a multicenter, open-label phase 2 study. Blood 2012;120:686

11. Bekkenk MW, Geelen FA, van Voorst Vader PC, et al. Primary and secondary cutaneous CD30(+) lymphoproliferative disorders: a report from the Dutch Cutaneous Lymphoma Group on the long-term follow-up data of 219 patients and guidelines for diagnosis and treatment. Blood 2000;95:3653-3661.

12. Woo DK, Jones CR, Vanoli-Storz MN, et al. Prognostic factors in primary cutaneous anaplastic large cell lymphoma: characterization of clinical subset with worse outcome. Arch Dermatol 2009;145:667-674.

13. Bareau B, Rey J, Hamidou M, et al. Analysis of a French cohort of patients with large granular lymphocyte leukemia: a report on 229 cases. Haematologica 2010;95:1534-1541.

14. Lamy T, Loughran TP Jr. How I treat LGL leukemia. Blood 2011;117:2764-2774. 